\title{
Tuberculosis - An Atypical Presentation
}

\author{
A Elmorsy, ${ }^{1, *}$ A. Clark-Morgan ${ }^{2}$, G. Shergill ${ }^{2}$ \\ ${ }^{1}$ Department of Trauma and Orthopaedics, Royal Surrey County, Guildford, UK \\ ${ }^{2}$ Department of Trauma and Orthopaedics, Salisbury District Hospital, Salisbury, UK \\ *Corresponding author: ahmedelmorsy@doctors.org.uk
}

Received March 22, 2015; Revised April 12, 2015; Accepted April 23, 2015

\begin{abstract}
Although endemic to the United Kingdom in the 19th and 20th centuries, cases of Tuberculosis have increased in the last twenty years making it an important differential diagnosis to consider. The recurrence of this almost extinct disease is thought to be a result of increasing prevalence of immune system deficiency, particularly Acquired Immunodeficiency Syndrome (AIDS). Musculoskeletal tuberculosis, particularly of a joint, remains a rare condition - accounting for less than $5 \%$ of cases of tuberculosis. Symptoms are mild and/or non-specific, making delayed or missed diagnosis a common problem. Here we present the unusual case of tuberculosis of the elbow to maintain consideration of tuberculosis as a differential diagnosis, even in cases with no strong suggestive medical history.
\end{abstract}

\section{Keywords: tuberculosis, elbow joint, musculoskeletal tuberculosis}

Cite This Article: A Elmorsy, A. Clark-Morgan, and G. Shergill, "Tuberculosis - An Atypical Presentation." American Journal of Medical Case Reports, vol. 3, no. 5 (2015): 146-147. doi: 10.12691/ajmcr-3-5-8.

\section{Case Report}

A 37 year old, right-handed, gentleman of Asian origin presented to the emergency department as a referral from his General Practitioner with an abscess of the left elbow and possible septic arthritis. His main complaints were six months of intermittent pain and swelling of the left elbow. Three days prior to presentation he developed a sudden onset of pain and gradually increasing swelling of the lateral aspect of the elbow. One month prior to this, he had developed a swelling of the medial aspect of the left upper arm which burst spontaneously. This continued to drain a yellowish discharge, however he sought no medical attention. One year before, he had a similar swelling and underwent brief hospital admission with no operative intervention. He had a dry cough for the week prior to admission. He denied night sweats, fever, loss of weight and anorexia. He had no known Tuberculosis contact and denied being treated for pulmonary tuberculosis in the past. He has no relevant past medical history and is unemployed. He has been living in the UK for fourteen years with no recent travels abroad.

On admission, he was unwell with a temperature of 38.2 degrees Celsius and tachycardia. There was a localized, warm, tender and fluctuant swelling in the anterolateral aspect of the left elbow, measuring $3 \times 3 \mathrm{~cm}$. It was surrounded by an area of cellulitis, extending across the forearm to the dorsal aspect of the hand. Elbow movements were restricted to 30 - 60 degrees of flexion and zero degrees supination-pronation. On the medial aspect of the distal third of the left upper arm there were two sinuses, draining scanty yellowish discharge with no surrounding cellulitis - suggesting chronicity. Admission blood results revealed Hemoglobin 119 g/L (N:138-128 $\mathrm{g} / \mathrm{L}$ ), Leucocyte $11200 / \mathrm{mm}^{3}$ (N:4500-10000), Neutrophil 9400 (N: 2500-7000) and C-reactive protein 154. Radiographs (Figure 1 \& Figure 2) showed irregular lucency in the humeral epicondyles, widening of the joint space and posterior subluxation of the olecranon process indicating joint effusion.

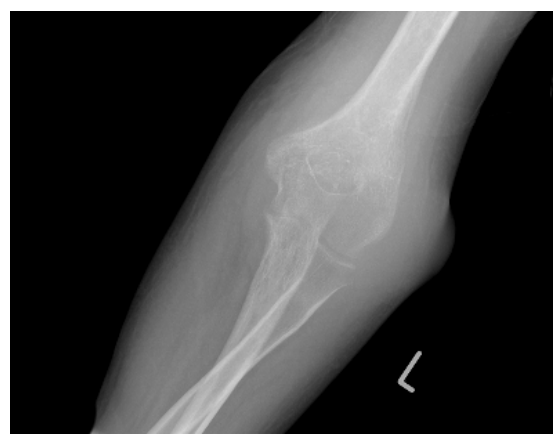

Figure 1. X-ray Anteroposterior view of the left elbow showing Juxsta articular Osteopaenia

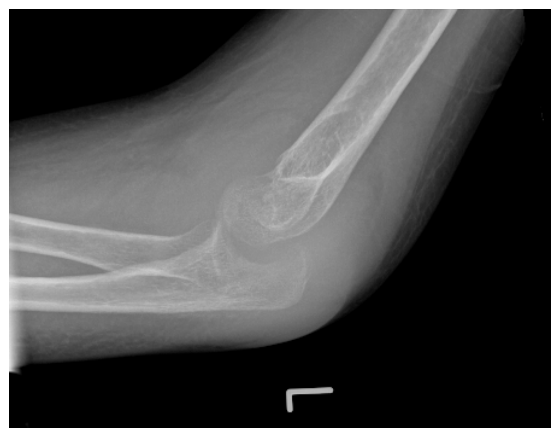

Figure 2. X-ray lateral view of the left elbow showing posterior subluxation of the olecranon 
Emergency incision and drainage of the abscess revealed pus and involvement of the joint space. Samples of pus and infected tissues, including synovial membrane, were sent for microbiological examination - comprising gram staining, Ziehl Neelsen staining, microscopy, culture and sensitivity. The wound was thoroughly debrided, washed out, packed and left open. The same was carried out for the sinuses on the medial aspect of the arm. Broad spectrum antibiotic therapy of Flucloxacillin and Benzyl Penicillin was commenced post-operatively. After fortyeight hours a second debridement was carried out and a vacuum dressing applied with the input of the Plastic Surgery team. A total of five debridement and washout procedures were carried out. The Ziehl Neelsen stain revealed acid fast bacilli and Mycobacterium tuberculosis were isolated on culture. Polymerase Chain Reaction (PCR) was positive for Mycobacterium Tuberculosis. However, HIV 1/2 Antigen/Antibody tests were negative. The cultures also grew Staphylococcus Aureus and Coliforms. Chest radiographs showed scattered nodular changes with superior mediastinal widening, confirmed later by CT to be due to adenopathy and small parenchymal changes.

After obtaining an input from the Respiratory physicians, Quadruple antibiotic therapy was commenced, comprising Rifampicin, Isoniazid, Pyrazinamide and Ethambutol in addition to the original Flucloxacillin and Benzyl Penicillin. The wound healed by secondary intention and the elbow ankylosed at 90 degrees of flexion with only 10 degrees of pronation and supination.

\section{Discussion}

As an endemic disease of urban poverty in the 19th and early 20th centuries, Tuberculosis caused millions of deaths and was only really halted by the discovery of antibiotics. Cases are again increasing and important factors are thought to be immigration from endemic countries, immunodeficient conditions including Acquired Immunodeficiency Syndrome (AIDS), alcoholism and drug addiction and intra-articular steroid injection [1,2]. Tuberculosis of the musculoskeletal system is a rare condition and accounts for less than $5 \%$ of its total infections, commonly affecting the spine, sacro-iliac, hip and knee joints. Tuberculosis of the elbow, however, accounts for less than $5 \%$ of musculoskeletal cases $[3,4,5]$.

Hematogenous spread of the tubercle bacillus from a pulmonary origin and to a lesser extent through lymphatic or direct spread leads to arthritis. The synovial membrane mounts an inflammatory reaction followed by granulation and pannus formation, eroding the cartilage and bone in a slow demineralization process. This early infection is nonpyogenic, so no proteolytic enzymes are formed, resulting in preservation of the joint space as the articular cartilage is spared until later in the disease process when secondary pyogenic infection may develop [2].

Symptoms of TB arthritis are always subtle and the delay between onset of symptoms and diagnosis ranges from 5-47 months [1]. Patients usually present with insidious onset pain, sometimes chronic, swelling, decreased range of movement and perhaps the usual systemic symptoms. Radiography is of little help in early disease, but as pyogenic infection develops, progressive Tuberculous disease is visible as the Phemister triad juxta-articular osteopenia or osteoporosis, narrowed joint space and peripheral osseous lesions [6]. Later in the disease process joints develop fibrous ankylosis. Chest radiographs are normal in approximately $50 \%$ of the patients, which could mistakenly lead to exclusion of the diagnosis and further delay.The diagnostic confusion is due to the similarity of disease with the more common pathologies like inflammatory arthropathy, septic arthritis, gout, pigmented villonodular synovitis and tumours [7]. Biopsy is the investigation of choice for diagnosis confirmation and may be image guided or surgical. The most accurate tissue samples are synovial membrane [6]. Before antibiotics, treatment for TB arthritis was immobilization, excision or arthrodesis. Now, the earlier the disease is detected and treated the better the outcome. Anti-tuberculosis drug therapy and early mobilization is the aim. Quadruple drug therapy currently comprises Rifampicin, Isoniazid, Ethambutol \& Pyrazinamide. Surgery is indicated for biopsy, synovectomy \& debridement in resistant cases and in late cases for arthrodesis [2].

For all cases of chronic joint pain Tuberculosis should remain a differential diagnosis, even in patients with irrelevant past medical history.

\section{References}

[1] Khan K, Muenning P, Bheta M, Zivin JG. Global drug-resistance patterns and the management of latent tuberculosis infection in immigrants to the United States. N Engl J Med 2002; 347:1850-9.

[2] Tuli SM. General principles of osteoarticular tuberculosis. Clin Orthop 2002: 11-19.

[3] Rahman MS, Brar R, Konchwalla A, Sala MJ. Pain in the elbow: a rare presentation of skeletal tuberculosis. J Shoulder Elbow Surg 2008; 17(1) : e19-21.

[4] Jung SS, Lee MK, Lim SH, Kwon YM, Choi SS Elbow pain proven to be tuberculous arthritis - a case report. Korean $J$ Anesthesiology 2010; 58: 211-14.

[5] Mandeep S Dhillon, Akshay Goel, Sharad Parghkar, Sameer Aggerwal, and Vikas Bachhal. Tuberculosis of the elbow: A clinicoradiological analysis. Indian J Orthop. 2012 Mar-Apr; 46(2): 200-205.

[6] Patel S, Collins DA, Bourke BE. Don't forget tuberculosis. Ann Rheum Dis.1995; 54: 174-5.

[7] Domingo A, Nomdedeu M, Tomas X, Garcia S. Elbow tuberculosis; And unusual location and a diagnostic problem. Arch Orthop Trauma Surg. 2005;125: 56-8. 